\title{
Court rules that withdrawal of life support is a plan of treatment requiring consent
}

$\mathrm{O}$ ntario doctors cannot withdraw life-sustaining treatment from patients without their consent or that of their substitute decision-makers, the province's Superior Court of Justice has ruled.

Physicians at the Sunnybrook Health Sciences Centre in Toronto, Ontario, had sought to withdraw mechanical ventilation from a 59-year-old man in a persistent, vegetative state without the consent of his substitute decision-maker.

The patient, Hassan Rasouli, a retired mechanical engineer, had undergone surgery at the hospital in October, 2010 to remove a benign tumour in his head. Following surgery, he had developed bacterial meningitis and ventriculitis, and had slipped into a coma, from which various experts said he would never emerge. He is being kept alive by a mechanical ventilator and feeding tube.

Dr. Brian Cuthbertson and Dr. Gordon Rubenfeld had sought to withdraw the life-sustaining equipment, place Rasouli on palliative therapy and transfer him to another facility. But Rasouli's spouse, Parichehr Salasel, a physician in Iran until the family emigrated to Canada in April 2010, objected, primarily on the grounds that withdrawal of life-sustaining treatment violated a tenet of the Shia Muslim faith that health care treatment should be provided until there are no signs of life.

Justice Susan Himel rejected the arguments of the physicians that withdrawal of life-sustaining treatment constituted a medical decision within their purview and that they were under no obligation to continue providing treatment because it would be of no benefit and would fall "outside the standard of care" (http://thaddeuspope.com /images/Rasouli_v._Sunnybrook_Ont ._2011_.pdf).

Withdrawal of life-sustaining equipment is part of the "plan of treatment,"

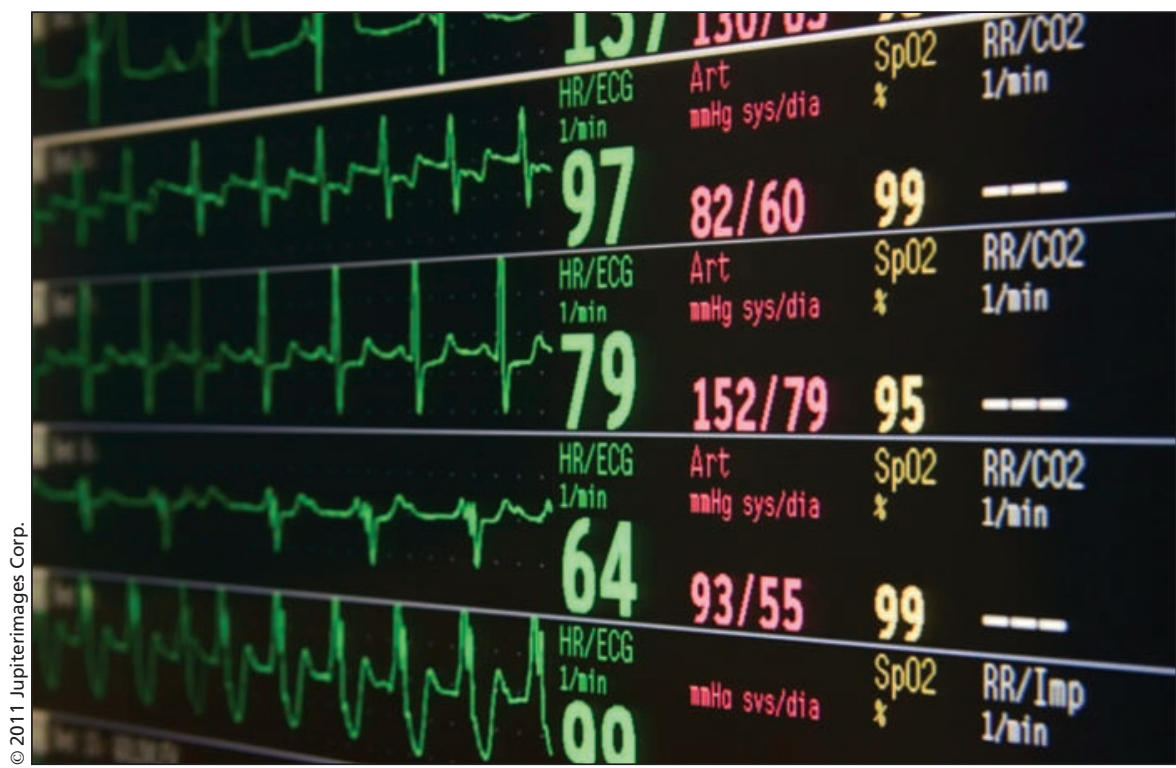

An Ontario judge rejected arguments that withdrawal of life-sustaining treatment in intensive care units constituted a medical decision falling strictly within the purview of physicians.

as defined by the province's Health Care Consent Act and therefore requires the consent of the patient or substitute decision-maker, Himel ruled.

Himel also rejected the physicians' argument that referral of such cases to the province's Consent and Capacity Board is "completely optional."

"If the respondents' interpretation of the HCCA [Health Care Consent Act] is accepted, no consistent rules regarding the withdrawal of life support in Ontario would exist. Whether or not substitute decision makers would be given an opportunity to consent would depend on the doctor in question, with recourse only being had to the CCB [Consent and Capacity Board] at the doctor's discretion."

Himel noted that doctors have recourse in cases in which they believe continuation of life-sustaining treatment provides no benefit to the patient and is not medically indicated. They can make their own case to the Consent and Capacity Board to have the substitute decision-makers stance set aside.
That provision also serves as a recourse for those who would argue that continuation of life-sustaining treatment constitutes an unnecessary drain on the finite financial resources of intensive care units, the judge added.

Pediatric intensivist Dr. Karen Choong calls the ruling "a very sensible one. There is good justification that consent is required for WLS [withdrawal of life support] as it is for other treatment."

Adult intensivist Dr. Claudio Martin concurs. "Unless we want to be barbaric and not provide palliative symptom management, withdrawal is really a replacement of one treatment plan with a new one and therefore requires consent."

The decision is being appealed by Cuthbertson and Rubenfeld, both of whom declined to be interviewed, as did the College of Physicians and Surgeons of Ontario. - Andrew B. Cooper MD, Paula Chidwick and Robert Sibbald, Toronto, Ont.

CMAJ 2011. DOI:10.1503/cmaj.109-3855 